\title{
The Statistical Properties of the Probabilistic Distributions Generated by Gamma Statistics
}

\author{
Oleg V. Chernoyarov ${ }^{1,2}$, Alexandra V. Salnikova ${ }^{2,3^{*}}$ and Vladimir I. Kostylev ${ }^{4}$ \\ ${ }^{1}$ Russia, 111250, Moscow, Krasnokazarmennaya st., 14, National Research University "Moscow Power Engineering Institute" \\ ${ }^{2}$ Russia, 634050, Tomsk, Lenin Avenue, 36, International Laboratory of Statistics of Stochastic Processes and Quantitative \\ Finance of National Research Tomsk State University \\ ${ }^{3}$ Russia, 394026, Voronezh, Moscow Avenue, 14, Voronezh State Technical University \\ ${ }^{4}$ Russia, 394018, Voronezh, Universitetskaya sq., 1, Voronezh State University \\ ${ }^{*}$ Corresponding author
}

\begin{abstract}
We show that hyperexponential and hyper-Erlang distributions reproduce themselves by composition. We find new formulas for probability densities and distribution functions of Gamma statistics sums.
\end{abstract}

Keywords-composition of statistics; replicating statistics; exponential, Gamma, Erlang distribution.

\section{INTRODUCTION}

Gamma distribution [1-3] is one of the most common probabilistic distributions in the statistical radio physics and radio engineering $[4,5]$. Particularly, by means of Gamma distribution, we can describe the effects at the outputs of the typical signal processing systems receiving the discrete Gaussian processes [5], multifrequency signals [6], radiation from complex radio sources [7], etc. The statistical properties of Gamma distribution can be described by means of the probability density

$$
w_{\gamma}(x ; a, A)=1(x) a^{-A} x^{A-1} \exp (-x / a) / \Gamma(A),
$$

where $a$ is the first [3] (scale [1]) parameter, $A$ is the second [3] (mean [1]) parameter of Gamma distribution; $1(x)= \begin{cases}1, & x \geq 0, \\ 0, & x<0\end{cases}$ is the Heaviside function; $\Gamma(A)=\int_{0}^{\infty} y^{A-1} \exp (-y) d y$ is the Gamma function [8]. Generally, both parameters of Gamma distribution are real.

The special case (under $A=1$ ) of Gamma distributions is the exponential distribution. The probability density of the exponential distribution has the form of [2]

$$
w_{e}(x ; a)=1(x) \exp (-x / a) / a \text {. }
$$

As another special case, the Erlang distribution can be introduced with the probability density [3]

$$
w_{E}(x ; a, K)=1(x) a^{-K} x^{K-1} \exp (-x / a) /(K-1) !
$$

which is Gamma distribution under integer values of the second parameter. The sums of squared absolute values of independent complex Gaussian variables (statistics [1]) with zero mean values and identical dispersions obey the Erlang distribution. Thus, the Erlang distribution is also frequently found in the reliability theory $[1,2]$.

Generalization of the exponential distribution is the hyperexponential distribution [2] with the probability density

$$
W_{e}(x ; M, \vec{\alpha}, \vec{a})=\sum_{m=1}^{M} \alpha_{m} w_{e}\left(x ; a_{m}\right),
$$

where $M$ is an integer parameter of the hyperexponential distribution, while $\vec{\alpha}=\left\{\alpha_{1}, \alpha_{2}, \ldots, \alpha_{M}\right\} \quad$ and $\vec{a}=\left\{a_{1}, a_{2}, \ldots, a_{M}\right\} \quad$ are real parameters of the hyperexponential distribution. Similarly, the hyper Gamma and hyper-Erlang distributions can be introduced with the probability densities

$$
\begin{aligned}
& W_{\gamma}(x ; M, \vec{\alpha}, \vec{a}, \vec{A})=\sum_{m=1}^{M} \alpha_{m} w_{\gamma}\left(x ; a_{m}, A_{m}\right) \text { and } \\
& \quad W_{E}(x ; M, \vec{\alpha}, \vec{a}, \vec{K})=\sum_{m=1}^{M} \alpha_{m} w_{E}\left(x ; a_{m}, K_{m}\right),
\end{aligned}
$$

correspondingly. Here $\vec{A}=\left\{A_{1}, A_{2}, \ldots, A_{M}\right\} \quad$ and $\vec{K}=\left\{K_{1}, K_{2}, \ldots, K_{M}\right\}$.

It is well known that some statistical distributions (such as normal, chi-square, binomial, Poisson) reproduce themselves by composition. Gamma distribution has a reproducing property by the second parameter only [3]: if two independent Gamma statistics have identical first parameters, then their sum also has Gamma distribution. The aim of the present paper is to define the statistical properties of the sums of two independent Gamma statistics under arbitrary parameter values as well as some sums related to them. 


\section{EXPONENTIAL STATISTICS}

The probability density $w_{e e}(x ; a, b)$ of the sum of two independent exponential statistics with the parameters $a$ and $b$ can be found as the convolution of two probability densities of the form (2). As a result, we obtain

$$
w_{e e}(x ; a, b)=a w_{e}(x ; a) /(a-b)+b w_{e}(x ; b) /(b-a) .
$$

We then compare Eqs. (4) and (6) and see that the sum of the two independent exponential statistics has the hyperexponential distribution. It is simple to show also that the distribution function

$$
f_{e e}(x ; a, b)=1-a^{2} w_{e}(x ; a) /(a-b)-b^{2} w_{e}(x ; b) /(b-a)
$$

corresponds to the probability density (6).

\section{HYPEREXPONENTIAL STATISTICS}

It is obvious that the probability density $W_{e e}(x ; M, \vec{\alpha}, \vec{a}, N, \vec{\beta}, \vec{b})$ of the sum of two independent hyperexponential statistics with the parameters $\{M, \vec{\alpha}, \vec{a}\}$ and $\{N, \vec{\beta}, \vec{b}\}$ is the hyperexponential probability density, namely,

$$
\begin{gathered}
W_{e e}(x ; M, \vec{\alpha}, \vec{a}, N, \vec{\beta}, \vec{b})=\sum_{m=1}^{M} \alpha_{m} \sum_{n=1}^{N} \beta_{n} w_{e e}\left(x ; a_{m}, b_{n}\right)= \\
=\sum_{m=1}^{M} A_{m} w_{e}\left(x ; a_{m}\right)+\sum_{n=1}^{N} B_{n} w_{e}\left(x ; b_{n}\right),
\end{gathered}
$$

where $A_{m}=\alpha_{m} a_{m} \sum_{n=1}^{N} \frac{\beta_{n}}{a_{m}-b_{n}}$ and $B_{n}=\beta_{n} b_{n} \sum_{m=1}^{M} \frac{\alpha_{m}}{b_{n}-a_{m}}$. Thus, it can be argued that the set of the hyperexponential probability densities is closed with respect to the convolution operation. That means that hyperexponential probability distribution replicates itself by composition.

\section{ERLANG AND EXPONENTIAL STATISTICS}

By calculating the convolution of the probability densities (2) and (3), we find the expression for the probability density $W_{E e}(x ; a, K, b)$ of the sum of independent Erlang and exponential statistics that appear to have the form of

$$
\begin{gathered}
W_{E e}(x ; a, K, b)= \\
=\left(\frac{b}{b-a}\right)^{K}\left[w_{e}(x ; b)+\frac{a}{a-b} \sum_{l=1}^{K}\left(\frac{b-a}{b}\right)^{l} w_{E}(x ; a, l)\right],
\end{gathered}
$$

where $a$ and $K$ are the Erlang statistics parameters, $b$ is the exponential statistics parameter.
We compare Eqs. (5) and (8) and see that the sum of independent Erlang and exponential statistics obeys hyperErlang distribution. The distribution function corresponding to the probability density (8) takes the form of

$$
\begin{gathered}
f_{E e}(x ; a, K, b)=1-[b /(b-a)]^{K} \times \\
\times\left\{b w_{e}(x ; b)+a \sum_{l=1}^{K}\left[\left(\frac{b-a}{b}\right)^{K}-\left(\frac{b-a}{b}\right)^{l-1}\right] w_{E}(x ; a, l)\right\} .
\end{gathered}
$$

We can see that the formulas (6) and (7) can be obtained from Eqs. (8) and (9) as a special case while $K=1$.

Based on Eqs. (8) and (9), it is easy to write the expressions for the probability density and the distribution function of the sum of hyper-Erlang and hyperexponential statistics.

\section{GAMMA AND EXPONENTIAL STATISTICS}

The probability density of the sum of independent Gamma statistics with the parameters $a$ and $A$ and exponential statistics with the parameter $b$ can be found as the convolution of the probability densities (1) and (2) and it has the form of

$$
w_{\gamma e}(x ; a, A, b)=\left(\frac{b}{b-a}\right)^{A} w_{e}(x ; b) f_{\gamma}\left(x ; \frac{a b}{a-b}, A\right)
$$

Here $f_{\gamma}(x ; a, A)=1(x) \mathrm{P}(A, x / a)$ is the distribution function corresponding to the probability density $\mathrm{P}(t, z)=\frac{1}{\Gamma(t)} \int_{0}^{z} y^{t-1} \exp (-y) d y$ is the normalized incomplete Gamma function [8].

The distribution function corresponding to Eq. (10) can be presented in the form of

$$
f_{\gamma e}(x ; a, A, b)=f_{\gamma}(x ; a, A)-b w_{\gamma e}(x ; a, A, b),
$$

while the probability density and the distribution function of the sum of independent hyper Gamma statistics with parameters $\{M, \vec{\alpha}, \vec{a}, \vec{A}\}$ and hyperexponential statistics with the parameters $\{N, \vec{\beta}, \vec{b}\}$ have the appearance

$$
\begin{gathered}
W_{\gamma e}(x ; M, \vec{\alpha}, \vec{a}, \vec{A}, N, \vec{\beta}, \vec{b})= \\
=\sum_{n=1}^{N} \beta_{n} w_{e}\left(x ; b_{n}\right) \sum_{m=1}^{M} \alpha_{m}\left(\frac{b_{n}}{b_{n}-a_{m}}\right)^{A_{m}} f_{\gamma}\left(x ; \frac{a_{m} b_{n}}{b_{n}-a_{m}}, A_{m}\right),
\end{gathered}
$$




$$
\begin{gathered}
F_{\gamma e}(x ; M, \vec{\alpha}, \vec{a}, \vec{A}, N, \vec{\beta}, \vec{b})= \\
=\sum_{m=1}^{M} \alpha_{m}\left[f_{\gamma}\left(x ; a_{m}, A_{m}\right)-\sum_{n=1}^{N} \beta_{n} b_{n} w_{e \gamma}\left(x ; a_{m}, A_{m}, b_{n}\right)\right] .
\end{gathered}
$$

\section{ERLANG STATISTICS}

From [3], we know the expression for the characteristic function of the Erlang distribution

$$
\theta_{E}(\eta ; a, K)=(1-i \eta a)^{-K}
$$

where $i=\sqrt{-1}$ is the imaginary unit.

The characteristic function of the sum of two independent statistics with the parameters $a, K$ and $b, L$ is the product of two characteristic functions of the form (13), that is,

$$
\theta_{E E}(\eta ; a, K, b, L)=(1-i \eta a)^{-K}(1-i \eta b)^{-L}
$$

In a specific case, from Eq. (14), we get

$$
\theta_{E E}(\eta ; a, K, a, L)=(1-i \eta a)^{-(K+L)}=\theta_{E}(\eta ; a, K+L),
$$

which means that the Erlang distribution is reproducible by the second parameter.

Generally, the right-hand member of Eq. (14) can be presented in the form of the sum of common fractions [9], namely,

$$
\begin{gathered}
\theta_{E E}(\eta ; a, K, b, L)= \\
=(-1)^{L} \frac{a^{L} b^{K}}{(L-1) !(b-a)^{K+L}} \sum_{l=1}^{K} \frac{(K+L-l-1) !}{(K-l) !}\left[\frac{b-a}{b(1-i \eta a)}\right]^{l}+ \\
+(-1)^{K} \frac{a^{L} b^{K}}{(K-1) !(a-b)^{K+L}} \sum_{l=1}^{L} \frac{(K+L-l-1) !}{(L-l) !}\left[\frac{a-b}{a(1-i \eta b)}\right]^{l} .
\end{gathered}
$$

We now transform Eq. (16) into

$$
\begin{gathered}
\theta_{E E}(\eta ; a, K, b, L)=C(a, K, b, L) \times \\
\times\left[\sum_{l=1}^{K} I_{l}(a, K, b, L) \theta_{E}(\eta ; a, l)+\sum_{l=1}^{L} J_{l}(a, K, b, L) \theta_{E}(\eta ; b, l)\right],
\end{gathered}
$$

where the designation are

$$
C(a, K, b, L)=\left(\frac{a}{a-b}\right)^{L}\left(\frac{b}{b-a}\right)^{K} \frac{(K+L-2) !}{(K-1) !(L-1) !}
$$

$$
\begin{aligned}
& I_{l}(a, K, b, L)=\left(\frac{b-a}{b}\right)^{l} \frac{(1-K)_{l-1}}{(2-K-L)_{l-1}}, \\
& J_{l}(a, K, b, L)=\left(\frac{a-b}{a}\right)^{l} \frac{(1-L)_{l-1}}{(2-K-L)_{l-1}}
\end{aligned}
$$

and $(a)_{m}=a(a+1) \ldots(a+m-1)$ is the Pochhammer symbol [8].

By applying the linear property of the Fourier transform, from Eq. (17), we can easily obtain the general expression for the probability density of the sum of two independent Erlang statistics:

$$
\begin{gathered}
w_{E E}(x ; a, K, b, L)=C(a, K, b, L) \times \\
\times\left[\sum_{l=1}^{K} I_{l}(a, K, b, L) w_{E}(x ; a, l)+\sum_{l=1}^{L} J_{l}(a, K, b, L) w_{E}(x ; b, l)\right],
\end{gathered}
$$

We compare Eqs. (5) and (18) and see that the sum of two independent Erlang statistics obeys the hyper-Erlang distribution. From this it follows that the sum of two independent hyper-Erlang statistics also has the hyper-Erlang distribution, that is, the hyper-Erlang distribution reproduces itself by composition.

From Eq. (18), it is easy to obtain the expression for the distribution function of the sums of two independent Erlang statistics:

$$
\begin{gathered}
f_{E E}(x ; a, K, b, L)=C(a, K, b, L) \times \\
\div\left[\sum_{l=1}^{K} I_{l}(a, K, b, L) f_{E}(x ; a, l)+\sum_{l=1}^{L} J_{l}(a, K, b, L) f_{E}(x ; b, l)\right],
\end{gathered}
$$

where

$$
f_{E}(x ; a, K)=1-\exp \left(-\frac{x}{a}\right) \sum_{l=0}^{K-1} \frac{1}{l !}\left(\frac{x}{a}\right)^{l}
$$

is the Erlang distribution function corresponding to the probability density (3). If we take into account Eq. (19), the expression (18) can be transformed into 


$$
\begin{gathered}
f_{E E}(x ; a, K, b, L)=1(x)\{1-C(a, K, b, L)+ \\
+\left[\exp \left(-\frac{x}{a}\right) \sum_{l=1}^{K} I_{l}(a, K, b, L) \sum_{m=0}^{l-1} \frac{1}{m !}\left(\frac{x}{a}\right)^{m}+\right. \\
\left.\left.+\exp \left(-\frac{x}{b}\right) \sum_{l=1}^{L} J_{l}(a, K, b, L) \sum_{m=0}^{l-1} \frac{1}{m !}\left(\frac{x}{b}\right)^{m}\right]\right\} .
\end{gathered}
$$

\section{GAMMA AND ERLANG STATISTICS}

Let there be two independent statistics, one of which described by Gamma distribution with the parameters $a, A$ and the other - by Erlang distribution with the parameters $b, B$. Then the probability density of their sum is the convolution of the probability densities (1) and (3). After simple, but though cumbersome, transformations we obtain

$$
\begin{gathered}
w_{\gamma E}(x ; a, A, b, L)=\left(\frac{a}{b}\right)^{L} w_{\gamma}(x ; a, A+L) \times \\
\times_{1} F_{1}\left(L, A+L, \frac{x}{a}-\frac{x}{b}\right),
\end{gathered}
$$

where ${ }_{1} F_{1}(\alpha, \beta, z)=\sum_{m=0}^{\infty} \frac{(\alpha)_{m} z^{m}}{(\beta)_{m} m !} \quad$ is $\quad$ the confluent hypergeometric function (Kummer's function) [8].

The distribution function corresponding to Eq. (20) can be presented in the form of the finite sum:

$$
f_{\gamma E}(x ; a, A, b, L)=f_{\gamma}(x ; a, A)-b \sum_{l=1}^{L} w_{\gamma E}(x ; a, A, b, l) .
$$

In the specified case, when $L=1$, we obtain the expression (10) from Eq. (20) as well as the expression (11) from Eq. (21). Thus, the expression for the distribution function of the sum of hyper Gamma and hyper-Erlang statistics generalizes the formula (12).

\section{GAMMA STATISTICS}

The probability density of the sum of two independent Gamma statistics is determined by the formula similar to Eq. (20), namely,

$$
\begin{gathered}
w_{\gamma \gamma}(x ; a, A, b, B)=\left(\frac{a}{b}\right)^{B} w_{\gamma}(x ; a, A+B) \times \\
\times_{1} F_{1}\left(B, A+B, \frac{x}{a}-\frac{x}{b}\right) .
\end{gathered}
$$

As ${ }_{1} F_{1}(\alpha, \beta, 0)=1$ irrespective of $\alpha$ and $\beta$, from Eq. (22) it follows that

$$
w_{\gamma \gamma}(x ; a, A, a, B)=w_{\gamma}(x ; a, A+B)
$$

Implication of Eq. (23) is analogous to the meaning of Eq. (15): under $a=b$ (and only in this case) the sum of two independent Gamma statistics has Gamma distribution.

For the distribution function of the sum of two independent Gamma statistics, we managed to get the integral expression only, in the form of

$$
\begin{gathered}
f_{\gamma \gamma}(x ; a, A, b, B)=\frac{1}{a} w_{\gamma}(x ; a, A) \times \\
\times \int_{0}^{1}(1-y)^{A-1} f_{\gamma}(x y ; b, B) \exp \left(\frac{x y}{b}\right) d y .
\end{gathered}
$$

It should be noted that the limits of integration and the subintegral function in Eq. (24) are such that the numerical computation by this formula is not difficult.

\section{CONCLUSION}

Thus, we have found the new expressions for the sums of two independent random variables which obey Gamma, exponential or Erlang distribution. We can show that the set of hyperexponential probability densities, as well as the set of hyper-Erlang probability densities, are closed with respect to the convolution operation, while the set of hyper Gamma probability densities does not possess this property.

\section{ACKNOWLEDGMENT}

This study was financially supported by the Russian Science Foundation (research project No. 17-11-01049).

\section{REFERENCES}

[1] W. Feller, An Introduction to Probability Theory and Its Applications, Vol. 2. New York, Wiley, 1971.

[2] V.S. Korolyuk, N.I. Portenko, A.V. Skorokhod and A.F. Turbin, Handbook on Probability Theory and Mathematical Statistics [in Russian]. Moscow, Mir, 1984.

[3] K.O. Bowman and L.R. Shenton, Properties of Estimators for the Gamma Distribution. New York, Marcel Dekker, 1988.

[4] H.L. van Trees, K.L. Bell and Z. Tian, Detection, Estimation, and Modulation Theory. Part I. Detection, Estimation and Filtering Theory. New York, Wiley, 2013.

[5] B.R. Levin, Theoretical Principles of Radioengineering Statistics. Virginia, Defense Technical Information Center, 1968.

[6] P.A. Bakut, I.A. Bolshakov, G.P. Tartakovsky, et. al., Problems of the Statistical Theory of Radar. Virginia, Defense Technical Information Center, 1964.

[7] V.I. Kostylev, "Detection of extended radio source in noise", Radiophysics and Quantum Electronics, vol. 42, pp. 352-356, April 1999.

[8] Handbook of Mathematical Functions with Formulas, Graphs and Mathematical Tables, Edited by M. Abramowitz and I. A. Stegun, National Bureau of Standards, Applied Mathematics Series 55, USA, 1964. 
[9] G.A. Korn and T.M. Korn, Mathematical Handbook for Scientists and Engineers: Definitions, Theorems, and Formulas for Reference and Review. New York, Dover Publications, 2000. 